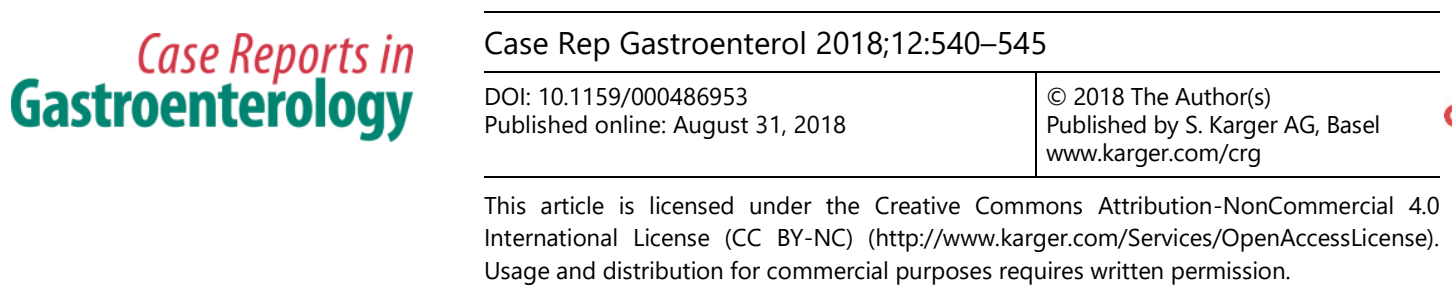

\title{
Successful Laparoscopy-Assisted Resection of the Descending Colon in a Patient with Multiple Large Renal Cysts and Stricture of the Colon due to Ischemic Colitis
}

\author{
Hiromichi Maeda ${ }^{a}$ Ken Okamoto ${ }^{a} \quad$ Tsutomu Namikawa $^{b} \quad$ Mai Shiga $^{b}$ \\ Kazune Fujisawa $^{b}$ Michiko Tadokoro $^{c}$ Kazuhiro Hanazaki ${ }^{b}$ \\ Michiya Kobayashi ${ }^{\mathrm{a}}$ \\ ${ }^{a}$ Cancer Treatment Center, Kochi Medical School Hospital, Nankoku, Japan; \\ ${ }^{b}$ Department of Surgery, Kochi Medical School, Kochi University, Nankoku, Japan; \\ 'Department of Radiology, Kochi Medical School, Kochi University, Nankoku, Japan
}

\section{Keywords}

Renal cyst · Laparoscopy · Colectomy · Colitis

\begin{abstract}
Large pathological structures in the abdominal cavity curb the application of laparoscopic surgery. This case report describes a successful laparoscopy-assisted colectomy for benign colon disease in a patient with multiple large renal cysts. An 82-year-old man was referred to our department for treatment of stenosis of descending colon secondary to ischemic colitis. An abdominal computed tomography revealed multiple large renal cysts occupying a large proportion of the peritoneal cavity. To minimize the postoperative recovery period, laparoscopic surgery was planned despite the renal cysts. After inserting access ports, the walls of the several renal cysts were fenestrated with an electronic scalpel and the serous fluid was aspirated to enable continuation of the laparoscopic colectomy. The left colon was mobilized and extracted through an incision at the umbilicus, and the affected part of the colon was resected safely.
\end{abstract}


The patient's postoperative course was uneventful, and the present case suggests that laparoscopy-assisted colectomy can be performed safely even in patients with multiple large renal cysts.

\section{Introduction}

Laparoscopic surgery for colorectal disease offers a shorter recovery period, less pain after surgery, and reduced morbidity compared with open surgery [1-3]. However, limited field of vision due to the narrow intra-abdominal space is a significant disadvantage of laparoscopic surgery $[1,4]$. For example, a very large abdominal mass makes it physically impossible for surgeons to perform laparoscopic surgery.

In this report, we describe a case of successful laparoscopy-assisted surgical resection of the descending colon in a patient with multiple large renal cysts. The renal cysts were fenestrated and the internal content was aspirated, enabling safe continuation of the laparoscopyassisted colectomy.

\section{Case Presentation}

An 81-year-old man was referred to our department for treatment of stenosis in the descending colon secondary to ischemic colitis. A colonoscopy and barium enema examination revealed that a long segment of the descending colon had a narrowed lumen (Fig. 1a). Blood tests revealed a slightly elevated serum creatinine level $(1.3 \mathrm{mg} / \mathrm{dL}$ [normal range, 0.6-1.1 $\mathrm{mg} / \mathrm{dL}]$ ). The plain abdominal computed tomography revealed multiple large bilateral renal cysts. The largest cyst was $12 \mathrm{~cm}$ in diameter, and there were more than 20 cysts in total (Fig. $1 \mathrm{~b}, \mathrm{c})$. Although the patient's family history was unremarkable, further testing was recommended to determine whether the patient had polycystic kidney disease. However, the patient and his family declined further testing after they were provided with information on polycystic kidney disease.

To treat the stenosis, laparoscopy-assisted colectomy was planned. Under general anesthesia, the first trocar for pneumatoperitoneum and the laparoscope was introduced through an incision at the umbilicus. Intra-abdominal pressure of $8 \mathrm{~mm} \mathrm{Hg}$ was created to enable observation of the peritoneal cavity. We observed no pathological ascites and no strong adhesion between the inner surface of the abdominal wall and the internal organs. To insert the surgical instruments, a 5-mm port was placed at the right upper abdomen and a 12-mm port was placed at the right lateral abdomen. The small intestine was relocated to the right side, with the left side of the body slightly elevated. The cysts of the left kidney were visible through the thin mesentery of the transverse and descending colon (Fig. 2a, b). Four large renal cysts of the left kidney were fenestrated with a monopolar scalpel, and the serous fluid of the cysts was aspirated. This created a larger working area for the surgery (Fig. 2c) and enabled observation of the splenic flexure.

The descending colon and distal part of the transverse colon were mobilized from the lateral side to the midline, and further fenestration and aspiration of renal cysts was performed as necessary. After mobilization of the descending colon and distal transverse colon was completed, a $3-\mathrm{cm}$ incision was made at the umbilicus and the colon was extracted through it. The region of interest was then resected and an anastomosis was completed using functional end-to-end anastomotic technique. The surgery took a total of $140 \mathrm{~min}$, and there 
was minimal estimated blood loss. The postoperative course was uneventful, and the patient was discharged 9 days after surgery. Inflammatory cell infiltration and fibrosis were present in the resected specimen, consistent with postischemic colitis.

\section{Discussion}

Ischemic colitis is caused by hypoperfusion of the colon, which can occur due to a variety of etiologies. Although ischemic colitis can occur in any part of the colon, involvement of the left colon is considered more common [5]. In most patients, ischemic colitis is reversible, and luminal narrowing of the affected colon is rare (although the incidence of luminal narrowing has not been quantified) [5, 6]. Stricture due to ischemic colitis is usually treated by surgical resection, as in this case.

Renal cysts are commonly detected on radiological imaging. Simple cysts can be present at birth [7], but most are acquired lesions [8]. The internal surface of a simple cyst is lined by a layer of flattened or cuboidal epithelial cells [9] and is surrounded by a thin layer of fibrous tissue. Simple cysts are generally solitary and asymptomatic [8, 9]. Occasionally, a cyst will cause abdominal pain, hydronephrosis, and abdominal distention, requiring aspiration of the cyst content or fenestration of the cyst wall [10]. While aspiration of the fluid content may be useful in terms of understanding the relationship between symptoms and the cyst, fenestration could be useful for permanent alleviation of symptoms [10]. In our patient, the walls of the cysts were not irregular and multilobulation was not observed, meaning that concurrent malignancy was unlikely [11]. Thus, fenestration of the cyst walls was considered an acceptable method for creating a sufficient intra-abdominal space for laparoscopic surgery.

To our knowledge, this is the first report of successful laparoscopy-assisted colectomy for colonic stenosis in a patient with multiple large renal cysts. Reducing the volume of the renal cysts by fenestration and aspiration of internal content enabled continuation of laparoscopic surgery. The benefits of laparoscopic surgery for patients are significant, and we believe that effort is therefore warranted to overcome obstacles when the procedure is rational and safe.

\section{Statement of Ethics}

Kochi Medical School Institutional Review Board (IRB) reviewed the protocol and judged that discussing this study (case report) in IRB is not necessary. The patient gave permission of the data usage for case presentation.

\section{Disclosure Statement}

The authors declare that there is no conflict of interest related to this case report.

\section{References}

1 Jones OM, Lindsey I, Cunningham C. Laparoscopic colorectal surgery. BMJ. 2011 Dec;343:d8029.

2 Tan JJ, Tjandra JJ. Laparoscopic surgery for ulcerative colitis - a meta-analysis. Colorectal Dis. 2006 Oct;8(8):626-36.

3 Schwenk W, Haase 0, Neudecker J, Müller JM. Short term benefits for laparoscopic colorectal resection. Cochrane Database Syst Rev. 2005 Jul;20(3):CD003145. 
4 Küper MA, Eisner F, Königsrainer A, Glatzle J. Laparoscopic surgery for benign and malign diseases of the digestive system: indications, limitations, and evidence. World J Gastroenterol. 2014 May;20(17):4883-91.

5 Zou X, Cao J, Yao Y, Liu W, Chen L. Endoscopic findings and clinicopathologic characteristics of ischemic colitis: a report of 85 cases. Dig Dis Sci. 2009 Sep;54(9):2009-15.

6 Brandt LJ, Feuerstadt P, Blaszka MC. Anatomic patterns, patient characteristics, and clinical outcomes in ischemic colitis: a study of 313 cases supported by histology. Am J Gastroenterol. 2010 0ct;105(10):224552.

7 Koutlidis N, Joyeux L, Méjean N, Sapin E. Management of simple renal cyst in children: french multicenter experience of 36 cases and review of the literature. J Pediatr Urol. 2015 Jun;11(3):113-7.

8 Chang CC, Kuo JY, Chan WL, Chen KK, Chang LS. Prevalence and clinical characteristics of simple renal cyst. J Chin Med Assoc. 2007 Nov;70(11):486-91.

9 Eknoyan G. A clinical view of simple and complex renal cysts. J Am Soc Nephrol. 2009 Sep;20(9):1874-6

10 Iannelli A, Fabiani P, Niesar E, Gigante M, Benizri EI, Amiel J, et al. Long-term results of transperitoneal laparoscopic fenestration in the treatment of simple renal cysts. J Laparoendosc Adv Surg Tech A. 2003 Dec;13(6):365-9.

11 Israel GM, Bosniak MA. An update of the Bosniak renal cyst classification system. Urology. 2005 Sep;66(3):484-8. 


\begin{tabular}{|c|c|c|}
\hline Case Reports in & Case Rep Gastroenterol 2 & $2: 540-545$ \\
\hline Gastroenterology & DOI: 10.1159/000486953 & $\begin{array}{l}\text { O } 2018 \text { The Author(s). Published by S. Karger AG, Basel } \\
\text { www.karger.com/crg }\end{array}$ \\
\hline
\end{tabular}
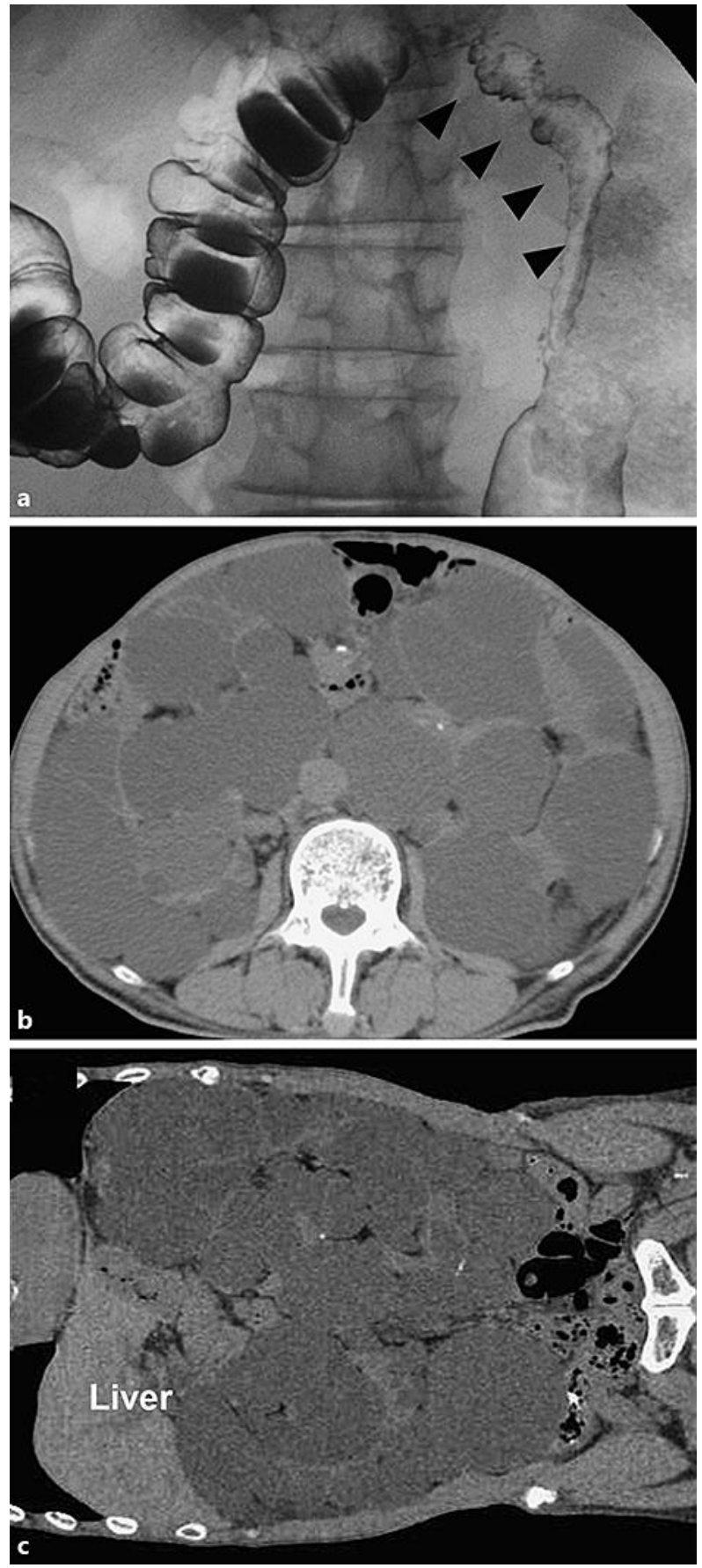

Fig. 1. Preoperative radiological examination. a Image from a barium enema examination, performed 2 months after the episode of ischemic colitis, revealing the development of stricture of the left colon that balloon dilation was unlikely to relieve. b, c Plain abdominal computed tomography images showing bilateral multiple renal cysts occupying a large proportion of the peritoneal cavity. No cysts were observed in other visceral organs, including the liver. b Horizontal plane. c Coronal plane. 


\begin{tabular}{lll} 
Case Reports in & \begin{tabular}{l} 
Case Rep Gastroenterol 2018;12:540-545 \\
\cline { 2 - 3 } Gal: 10.1159/000486953
\end{tabular} & $\begin{array}{l}\text { @ } 2018 \text { The Author(s). Published by S. Karger AG, Basel } \\
\text { www.karger.com/crg }\end{array}$ \\
\hline
\end{tabular}
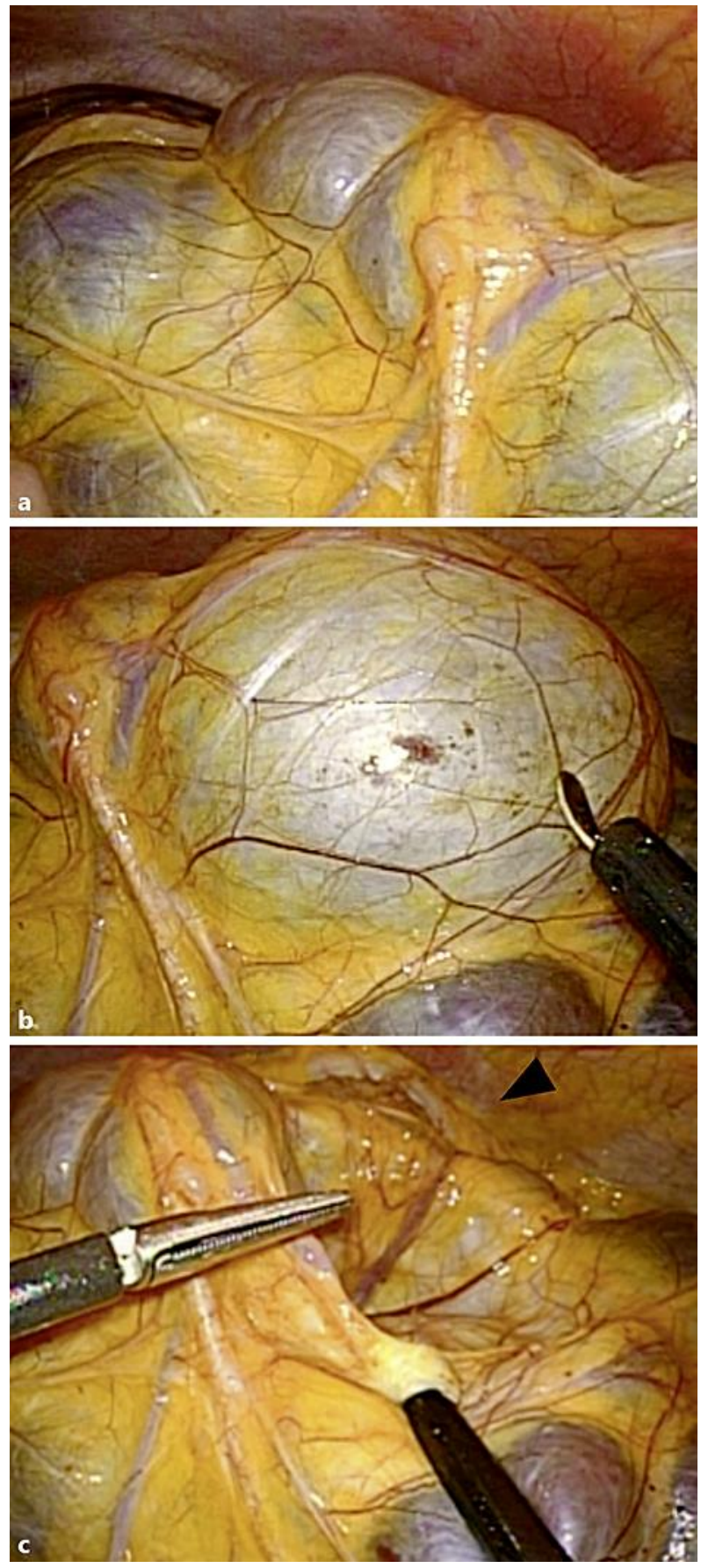

Fig. 2. Intraoperative findings. a The cysts are visible as white or purple protrusions under the thin mesentery of the colon. Observation of the left upper abdomen from the umbilicus is hindered by a large cyst. b To facilitate the surgical procedure, the walls of the cysts were fenestrated together with the mesentery. c After aspiration of the inner content of the cysts, the transverse colon is visible from the same position (arrowhead). 